\title{
A rare case of rapidly enlarging tracheal lobular capillary hemangioma presenting as difficult to ventilate acute asthma during pregnancy
}

\author{
Shivesh Prakash ${ }^{1 *}$, Shailesh Bihari ${ }^{2}$ and Ubbo Wiersema ${ }^{1}$
}

\begin{abstract}
Background: Lobular Capillary Hemangioma $(\mathrm{LCH})$ is a benign tumour that is known to be hormone responsive and have a relatively high incidence during pregnancy, the most common site being the gingival surfaces. A tracheal origin for this tumour is extremely rare, with no case reported so far in this patient population, and the only reported clinical presentation of tracheal LCH in the literature is with haemoptysis.

Case presentation: We describe a case of a 23-year-old known asthmatic who presented at 32 weeks gestation with life-threatening respiratory failure resembling acute severe asthma, requiring invasive ventilation which was extremely difficult. This was subsequently found to be due to a large tracheal LCH producing a ball-valve phenomenon and predominantly expiratory airflow limitation similar to acute asthma. The endotracheal tube was advanced past the lesion under bronchoscopic guidance, and urgent Caesarean section performed due to foetal distress. The tumour was subsequently debulked and the trachea stented, facilitated by bi-femoral veno-venous extra-corporeal membrane oxygenation with relatively low dose of heparin.
\end{abstract}

Conclusion: To our knowledge, this is the first report of a unique presentation and management of largest tracheal $\mathrm{LCH}$ so far occurring during pregnancy. Pulmonary and critical care physicians should be aware of this unique differential of refractory asthma, the aggressive nature of this benign tumour due to hormonal influences during pregnancy, and feasibility of using bi-femoral veno-venous extra-corporeal membrane oxygenation with low dose heparin as a rescue, given the high risk of bleeding.

Keywords: Lobular Capillary Hemangioma, Asthma, Pregnancy, Veno-venous extra-corporeal membrane oxygenation

\section{Background}

Primary tracheal tumours are rare with an estimated incidence of about 2.7 new cases per million per year and are usually malignant in adults [1]. Lobular Capillary Haemangioma ( $\mathrm{LCH}$; pyogenic granuloma) is a benign tumour with a distinctive lobular arrangement of capillaries [2]. The usual sites for this tumour are the skin and the nasopharyngeal and oral mucosal surfaces. A tracheal origin for this tumour is, however, exceedingly rare, with the literature limited to only a few case reports $[3,4]$. Hence, little is known about the presentation, behaviour, and management of tracheal LCH. This tumour is hormone responsive and consequently has a

\footnotetext{
* Correspondence: shivesh.prakash@health.sa.gov.au

${ }^{1}$ Department of Intensive care, Flinders Medical Centre, Bedford Park,

5042 South Australia, Australia

Full list of author information is available at the end of the article
}

relatively higher incidence, recurrence, and growth rate during pregnancy [5]. However, a tracheal origin for $\mathrm{LCH}$ has not been reported in this patient population so far. Also, none of the case reports have clearly revealed the recurrent nature and rate of growth of tracheal $\mathrm{LCH}$. We present the case of a 23-year-old pregnant woman with asthma who presented with tracheal LCH masquerading as acute asthma, posing both a diagnostic and therapeutic challenge.

\section{Case presentation}

A 32 weeks pregnant, 23-year-old known asthmatic woman presented to a peripheral hospital with acute respiratory failure during winter. Cold air was a known precipitant for her asthma. She was intubated and ventilated for presumed severe acute asthma with refractory bronchospasm. Following intubation she was found to 
be extremely difficult to ventilate and was retrieved to our intensive care unit for further management.

She had widespread, faint, prolonged monophonic wheeze on auscultation with no evidence of pneumothorax. Mechanical ventilation in volume control mode was commenced with an inspired oxygen fraction of 1.0, external Positive End Expiratory Pressure (PEEP) $0 \mathrm{cmH}_{2} \mathrm{O}$, respiratory rate six breaths per minute, a tidal volume of $300 \mathrm{ml}$, and inspiratory flow rate of $30 \mathrm{~L} / \mathrm{min}$. However, high peak airway pressures truncated each breath. Intrinsic PEEP was measured as $30 \mathrm{cmH}_{2} \mathrm{O}$, with evidence of dynamic hyperinflation and accompanying hemodynamic instability despite inspiratory to expiratory ratio of close to $1: 16$. An arterial blood gas sample revealed severe respiratory acidosis $(\mathrm{pH}$ of 6.92 , $\mathrm{PaO}_{2} 116 \mathrm{mmHg}$, and $\mathrm{PaCO}_{2} 143 \mathrm{mmHg}$ ). There was evidence of foetal distress on Cardiotocograph (CTG). Bronchoscopy demonstrated a pedunculated tumour arising from the posterior tracheal wall, extending from the tip of the Endotracheal Tube (ET) and measuring approximately $4 \mathrm{~cm}$ by $2 \mathrm{~cm}$. The tumour acted as a ball valve to cause severe expiratory airflow obstruction (Figure 1; Additional file 1: Video 1). The bronchoscope was advanced beyond the lesion and the ET advanced over the bronchoscope to bridge the lesion, resulting in dramatic improvements in lung mechanics and haemodynamics. Urgent Caesarean section was performed due to persistent decelerations on CTG, with birth of a healthy baby. Access to her previous records revealed that she previously presented with haemoptysis at 16 weeks of the current gestation. At this time she had a small polyp $(\sim 0.5 \mathrm{~cm})$ in the same tracheal location, which had been excised and diagnosed as LCH.

The following day, tumour debulking was attempted. In view of the high risk of major haemorrhage and hypoxemia, precautionary cannulation for veno-venous Extra-Corporeal Membrane Oxygenation (ECMO) was established via a bi-femoral approach. Surgical debulking was complicated by significant haemorrhage causing desaturation $\left(\mathrm{SpO}_{2}<80 \%\right)$. She was promptly commenced on ECMO with recovery of arterial oxygen saturation and minimal disruption to surgery. A small bolus of heparin (2000 units) was given intravenously just prior to connection of the ECMO circuit, but no further heparin was administered until after surgery. On return to the ICU, a low dose heparin infusion (10 units $/ \mathrm{kg} /$ hour) was used to maintain ECMO circuit patency. The histopathology revealed recurrent LCH (Figure 2), with intact overlying epithelium (Arrow) and lobular arrangements (*) of proliferating capillaries (horizontal arrow) in an edematous fibro-myxoid stroma, containing extravasated red blood cells (vertical arrow). There was intense staining with CD31 Immunohistochemical staining highlighting proliferating endothelial cells and vascular origin of tumor. Her trachea was subsequently stented and ECMO was discontinued. She made a full recovery.

\section{Discussion}

Obstructing airway lesions are recognised in the differential diagnosis of refractory asthma. Primary tracheal tumours are rare with an estimated incidence of about 2.7 new cases per million per year and are usually malignant in adults [1]. Amongst all tracheal tumours, LCH is exceedingly rare with only few case reports in the literature $[3,4]$. The usual sites for this tumour are the skin and the nasopharyngeal and oral mucosae. The cause of tracheal obstruction in this case was found to be a large $\mathrm{LCH}$. To our knowledge, this is the largest case of tracheal $\mathrm{LCH}$ reported to date. $\mathrm{LCH}$ occurs in patients of all ages with a peak incidence in the second and third decades of life [6]. Mucosal LCH is more common in adult women than in men or children [7].

Microscopically, $\mathrm{LCH}$ has a distinctive lobular arrangement of capillaries of varying calibre embedded in a fibromyxoid matrix without atypical mitoses [2]. Conventional

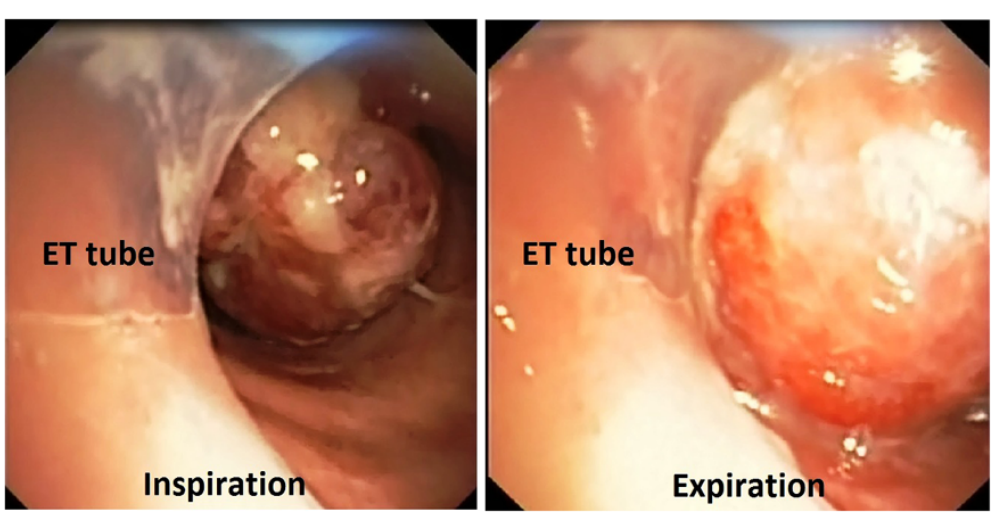

Figure 1 Bronchoscopic view at the edge of endotracheal tube. A highly vascular polypoidal lesion is seen, which causes a ball-valve phenomenon during respiration to generate severe compromise of expiratory airflow (Additional file 1: Video 1). 


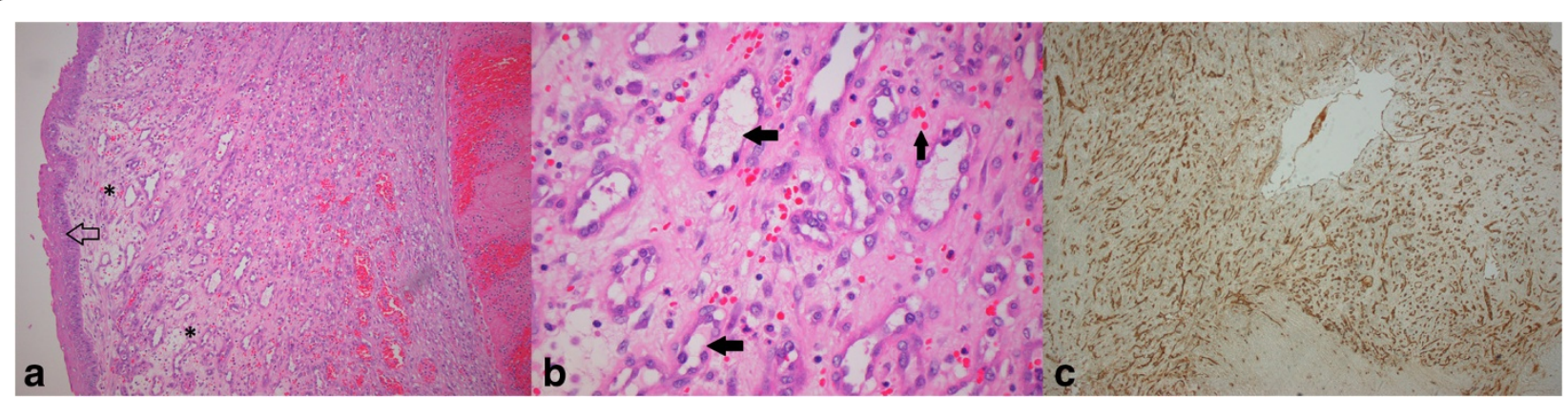

Figure 2 Histopathological features of Lobular capillary hemangioma. Hematoxylin and eosin stained sections at (a) x100 Magnification and (b) ×400 Magnification, and (c) CD31 Immunohistochemical staining, (Magnification x40).

granulation tissue is the most important histological differential diagnosis. Since LCH is most commonly found in locations prone to trauma such as skin, gingiva, nail beds, etc., finding ulceration and coexisting chronic inflammation in the stroma is fairly common. When inflammation is marked, the overall features show a close resemblance to granulation tissue, except for the presence of capillary lobules in the deeper dermis at the base of the lesion, lobular arrangements of capillaries instead of radial, and proliferating endothelial cells on immunohistochemical staining (e.g., with anti-CD31 antibodies in this case). The granulomatous appearance is highly variable depending on the location of the tumour and is frequent in cutaneous and mucosal locations, which are more prone to trauma. Besides histology, LCH differs from granulation tissue by its rapid growth, multiple occurrence, and frequent recurrence. The lesion tends to have a pedunculated macroscopic morphology, which along with its large size led to the ball-valve phenomenon and phasic airflow limitation in this case [2]. The pedunculated morphology is seen in most LCH occurring on the skin but only in 30 percent of mucosal $\mathrm{LCH}$, mainly in those involving the oral mucosa [2]. Such a presentation of LCH has not been reported before. The first presentation, however, was with haemoptysis and this is the most typical presentation apart from simple cough that has been reported in the literature.

The pathogenesis of this lesion is currently uncertain. Most theories on pathogenesis revolve around LCH as a hyperplastic, neovascular response to an angiogenic stimulus with imbalance of promoters and inhibitors [8]. Angiogenic growth factors such as vascular endothelial growth factor (VEGF) and decorin, transcription factors (pATF2 and pSTAT3), and signal transduction pathways (MAPK) are overexpressed in $\mathrm{LCH}$, but their exact role is undetermined $[9,10]$. There is a reported association between $\mathrm{LCH}$ and pregnancy ( $5 \%$ incidence), where it is termed granuloma gravidarum [5]. $\mathrm{LCH}$ are known to recur and grow rapidly during pregnancy, presumably due to responsiveness to progesterone, which appears to act via stimulation of angiogenic factors and inhibition of apoptosis [5,11]. This may explain the recurrent nature of the tumour and the rapidity of growth in the case discussed. Trauma has been reported as a trigger, although only 7 to $23 \%$ of patients with $\mathrm{LCH}$ report a previous injury at the site [12]. There was no history of airway instrumentation or foreign body aspiration prior to the bronchoscopy performed earlier in her gestation, when the tumour was already in situ.

The gingival surface is the most common site of $\mathrm{LCH}$ during pregnancy, with no cases of tracheal $\mathrm{LCH}$ reported during pregnancy to date. None of the case reports have clearly revealed the recurrent nature and rate of growth of tracheal LCH as they were already large, symptomatic, and requiring treatment at the time of diagnosis. The current knowledge about these features of this tumour mainly originates from literature on extra-tracheal sites of $\mathrm{LCH}$, particularly the skin. $\mathrm{LCH}$ rarely exceeds $2.5 \mathrm{~cm}$ in size and it usually reaches its full size within weeks or months, remaining static thereafter [13]. The LCH occurring in pregnant women usually regress after childbirth over a period of 6 to 18 months. A recurrence rate of $16 \%$ has been reported for oral $\mathrm{LCH}$ and is believed to result from incomplete excision, failure to remove etiological factors, or re-injury of the area [14]; the inability to remove aetiological factors is probably responsible for the higher recurrence rate observed during pregnancy. The case discussed is the first evidence to suggest that tracheal $\mathrm{LCH}$ can have high recurrence rate and rapid growth, justifying surveillance after initial treatment.

The mainstay of treatment, if symptomatic, is surgical debulking or excision using various methods [2-4,12]. The use of ECMO to facilitate tracheal surgery has previously been reported [15], and this case illustrates the feasibility of low dose heparinisation and bifemoral cannulation, which facilitated the rapid use of ECMO with minimal interruption to surgery. ECMO has not been utilised in the management in the previous case reports of tracheal LCH. ECMO has significant risks and cost 
implications and is not recommended as routine in every case of tracheal LCH. A few characteristics of this particular case, however, prompted consideration of use of ECMO, such as the large size of the tumour, its highly vascular nature due to the hormonal influence of pregnancy, and its proximity to the carina. Even then, ECMO was planned as a rescue and not as a routine first-line strategy to facilitate surgery.

\section{Conclusion}

Here we describe a rare differential of refractory asthma the largest $\mathrm{LCH}$ tumour reported to date, occurring at an exceedingly rare site and with rapid growth during pregnancy, possibly due to hormonal influence. Pulmonary and critical care physicians should be aware of this unique differential of refractory asthma, the association with pregnancy, and the feasibility of using bi-femoral veno-venous ECMO with low-dose heparin for its management.

\section{Consent}

Written informed consent was obtained from the patient for publication of this case report and any accompanying images. A copy of the written consent is available for review by the Editor of this journal.

\section{Additional file}

Additional file 1: Video 1. Bronchoscopic view at the edge of endotracheal tube. A highly vascular polypoidal lesion is seen, which causes a ball-valve phenomenon during respiration to generate severe compromise of expiratory airflow.

\section{Abbreviations}

CTG: Cardiotocograph; ET: Endotracheal tube; ECMO: Extra-corporeal membrane oxygenation; LCH: Lobular capillary hemangioma; PEEP: Positive end expiratory pressure.

\section{Competing interests}

The authors declare that they have no competing interests.

\section{Authors' contributions}

SP and SB analysed and interpreted the patient's data and drafted the manuscript. UW revised the clinical data and supervised the case report. All authors read and approved the final manuscript.

\section{Authors' information \\ SP and SB have MD in pulmonary medicine and currently hold Australian fellowship in intensive care. Their area of interest includes critical care aspects of pulmonary medicine. UW is a senior consultant in intensive care. He has expertise in the area of ECMO and has been the director of ECMO services at Flinders Medical Centre Intensive care.}

\section{Author details}

${ }^{1}$ Department of Intensive care, Flinders Medical Centre, Bedford Park, 5042 South Australia, Australia. ${ }^{2}$ Department of Critical Care Medicine, Flinders University, Bedford Park, 5042 South Australia, Australia.

Received: 20 July 2013 Accepted: 19 February 2014 Published: 10 March 2014

\section{References}

1. Lau CL, Patterson GA: Diagnosis and Management of Tracheal Neoplasms. In Cummings Otolaryngology Head and Neck Surgery. 4th edition. Edited by Flint PW. PA: Elsevier Mosby; 2005:2478-2480.

2. Mills SE, Cooper PH, Fechner RE: Lobular capillary hemangioma: the underlying lesion of pyogenic granuloma; a study of 73 cases from the oral and nasal mucous membranes. Am J Surg Pathol 1980, 4:470-479.

3. Irani S, Brack T, Pfaltz M, Russi EW: Tracheal lobular capillary hemangioma: a rare case of recurrent hemoptysis. Chest 2003, 123:2148e9.

4. Amy FT, Enrique DG: Lobular Capillary Hemangioma in the Posterior Trachea: A Rare Cause of Hemoptysis. [http://dx.doi.org/10.1155/2012/592524]

5. Sills ES, Zegarelli DJ, Hoschander MM, Strider WE: Clinical diagnosis and management of hormonally responsive oral pregnancy tumor (pyogenic granuloma). J Reprod Med 1996, 41:467-470.

6. Harris MN, Desai R, Chuang TY, Hood AF, Mirowski GW: Lobular capillary hemangiomas: an epidemiologic report, with emphasis on cutaneous lesions. J Am Acad Dermatol 2000, 42:1012.

7. Gordón-Núñez MA, De Vasconcelos Carvalho M, Benevenuto TG, Lopes MF, Silva LM, Galvão HC: Oral pyogenic granuloma: a retrospective analysis of 293 cases in a Brazilian population. J Oral Maxillofac Surg 2010, 68:2185.

8. Giblin AV, Clover AJ, Athanassopoulos A, Budny PG: Pyogenic granuloma the quest for optimum treatment: audit of treatment of 408 cases. J Plast Reconstr Aesthet Surg 2007, 60:1030.

9. Chen SY, Takeuchi S, Urabe K, Hayashida S, Kido M, Tomoeda H, Uchi H, Dainichi T, Takahara M, Shibata S, Tu YT, Furue M, Moroi Y: Overexpression of phosphorylated-ATF2 and STAT3 in cutaneous angiosarcoma and pyogenic granuloma. J Cutan Pathol 2008, 35:722.

10. Arbiser JL, Weiss SW, Arbiser ZK, Bravo F, Govindajaran B, Caceres-Rios H, Cotsonis G, Recavarren S, Swerlick RA, Cohen C: Differential expression of active mitogen-activated protein kinase in cutaneous endothelial neoplasms: implications for biologic behavior and response to therapy. J Am Acad Dermatol 2001, 44:193.

11. Yuan K, Wing LY, Lin MT: Pathogenetic roles of angiogenic factors in pyogenic granulomas in pregnancy are modulated by female sex hormones. J Periodontol 2002, 73:701-708.

12. Patrice SJ, Wiss K, Mulliken JB: Pyogenic granuloma (lobular capillary hemangioma): a clinicopathologic study of 178 cases. Pediatr Dermatol 1991, 8:267.

13. Bouquot JE, Nikai H: Lesions of the Oral Cavity. In Diagnostic Surgical Pathology of the Head and Neck. 1st edition. Edited by Gnepp DR. Philadelphia: WB Saunders; 2001:141-233.

14. Taira JW, Hill TL, Everett MA: Lobular capillary hemangioma (pyogenic granuloma) with satellitosis. J Am Acad Dermatol 1992, 27:297-300.

15. Smith IJ, Sidebotham DA, McGeorge AD, Dorman EB, Wilsher ML, Kolbe J: Use of extracorporeal membrane oxygenation during resection of tracheal papillomatosis. Anesthesiology 2009, 110:427-429.

doi:10.1186/1471-2466-14-41

Cite this article as: Prakash et al:: A rare case of rapidly enlarging tracheal lobular capillary hemangioma presenting as difficult to ventilate acute asthma during pregnancy. BMC Pulmonary Medicine 2014 14:41

\section{Submit your next manuscript to BioMed Central and take full advantage of:}

- Convenient online submission

- Thorough peer review

- No space constraints or color figure charges

- Immediate publication on acceptance

- Inclusion in PubMed, CAS, Scopus and Google Scholar

- Research which is freely available for redistribution 